УДК $550.3,550.31$

\title{
ОЦЕНКА ОБЪЕМОВ УГЛЕВОДОРОДНЫХ ГАЗОВ ГАЗОГИДРАТОВ АЗЕРБАЙДЖАНСКОГО СЕКТОРА ЮЖНОГО КАСПИЯ ПО СЕЙСМИЧЕСКИМ ДАННЫМ
}

\author{
Александр Владимирович Полетаев', \\ avo1@mail.ru \\ Елена Владимировна Полетаева', \\ yelenapv@mail.ru \\ Национальная Академия Наук Азербайджана, \\ AZ1000, Азербайджан, г. Баку, ул. Ф. Амирова, 9.
}

\begin{abstract}
Актуальность исследования заключается в изучении верхней части разреза глубоководной части Южного Каспия с целью выделения зон газогидратов по сейсмическим данным, оценки их мощности, а также подсчета объемов газа газогидратов. Исследование газогидратов Южного Каспия необходимо для достоверной оценки энергетического потенциала углеводородных газов Азербайджана. Результаты изучения газогидратов также могут быть применены для оценки роли газовых гидратов в приповерхностных слоях геосферы, особенно в связи с их возможным влиянием на экологию Каспийского моря, региона и глобальные климатические изменения.
\end{abstract}

Цель исследования заключается в выделении и оценке объемов углеводородных газов газогидратов глубоководной части Южного Каспия по сейсмическим данным.

Объектом исследования послужили 17 региональных сейсмических профилей и данные термодинамики региона. По двум площадям Южного Каспия использованы данные скоростного анализа. При интерпретации сейсмических профилей особое внимание уделено идентификации горизонтов, содержащих газогидраты.

Методы исследования основаны на наличии прямой связи между особенностями геологического строения зон газогидратов и распределением в среде физических параметров. Индикаторами газогидрата на временном разрезе могут служить аномальные изменения сейсмических характеристик волн: амплитуда, частота, фаза, скорости продольных и поперечных волн. Наиболее широко применяемым сейсморазведочным признаком газогидрата является наличие на сейсмических записях отражающего сейсмического горизонта BSR, а также ярких пятен. По этим критериям интерпретированы все временные разрезы и установлены верхняя и нижняя границы зон с отражениями, характерными для зон газогидратов. Интерпретация данных производилась с помощью лицензионного программного комплекса Kingdom Suite 2017.

В результате исследования построены карты нижней и верхней границ зон газогидратов, а также рассчитаны мощности отложений, содержащих газогидраты. Объемы пород, установленные по сейсмическим данным, позволили рассчитать объемы углеводородных газов, содержащихся в газогидратах. Максимальный объем газа в исследуемой зоне составляет 8,06 трил м газа. Также рассчитан объем газогидратов, содержащихся в породе в пределах структур. Ранжирование данных по объемам показало, что в южной части Каспия содержится максимальное количество газа. Исходя из вышеизложенного в глубоководной части Южного Каспия можно выделить две перспективно-газогидратные зоны: высокоперспективная зона в южной части; перспективная зона в северной глубоководной части. Перечисленные зоны накопления газогидратов обеспечат энергетическую безопасность Азербайджана на многие годы.

\section{Ключевые слова:}

Газогидраты, сейсмические профили, оценка, газ, Южный Каспий, углеводороды.

\section{Введение}

В Азербайджане первая крупная работа по изучению газогидратов Каспийского моря проведена в период с 1972 по 1986 гг. Анализ равновесных параметров давления и температуры показал, что зона газогидратообразования в условиях Каспийского моря существует ниже изобаты 250 м. В результате морских исследований на дне Каспийского моря открыты скопления газогидратов, названные Буздаг, Элм, и одно проявление газогидратов на валу Абиха. Установлено, что скопление Буздаг располагается на грязевом вулкане вала Шатского на глубинах 480-500 м. В 17-ти поднятых грунтовых колонках были обнаружены газогидраты в виде ледоподобных полупрозрачных метановых образований различной формы и размеров. Газогидратное скопление «Элм» располагается на глубинах 560 м. Протяженность около 4 км. В пяти из семи случаев колонки оказались газогидратоносными [1].
В работе [2] приведены результаты морских исследований, выполненных в 5 милях от газогидратопроявления Элм. В этой зоне проведено сейсмоакустическое профилирование серийной аппаратурой «Аквамарин» на частотах 200-800 Гц с глубиной просвечивания $\approx 700$ м от дна и разрешающей способностью $\approx 10$ м. По сейсмоакустической записи была выделена диапировая структура. В центральной части диапира наблюдалось характерное затухание акустического сигнала, что, вероятно, обусловлено газоносыщенностью отложений. Эта структура представляет собой усечено-конусовидное образование высотой 180 м и диаметром у основания приблизительно 2,5 км. В 16 поднятых грунтовых колонках обнаружены гидратосодержащие грязевулканические отложения.

В 2004 г. опубликована работа [3], основанная на изучении термобарических параметров ЮКВ, в которой были подтверждены выделенные ранее зоны гидратообразования, указанные [1, 2, 4] в 
1986 г. Работы проведены с использованием математического решения: уравнения изменения термического градиента в разрезе пород и уравнения равновесного стабильного существования гидрата в данной пористой среде. Исходя из анализа вышеуказанных данных автор прогнозирует наличие газогидратов в осадочных породах до глубин 3000 м, в зоне глубин моря - 1000 м [3].

Комплексирование сейсмических и термодинамических данных региона проведено группой исследователей [5] для площади Абшерон. Авторы рассмотрели два глубоководных 20 с профиля, пересекающих Южный Каспий, и выяснили, что газогидраты выявляются до 2 с. Они изучали газогидраты на глубинах воды от 200 до 715 м. На этих профилях выделены мелководные высокоскоростные аномалии $\left(V_{p}=2,1 \mathrm{~km} / \mathrm{c}, V_{s}=0,8 \mathrm{~km} / \mathrm{c}\right)$. Авторы связывают эти аномалии с газогидратами. Кровля газогидрата маркировалась сильным положительным отражением $\left(R_{c}=0,123\right)$, а подошва - высоким отрицательным отражением $\left(R_{c}=0,11\right)$. Из анализа термобарической модели авторы сделали вывод, что глубина залегания газогидратов в отложениях площади Абшерон может устанавливаться до $\approx 1350 \mathrm{M}$.

В 2012 г. опубликована книга [6], в которой приводится описание различных видов газовых гидратов с момента их образования, термодинамических образующих факторов и кинетики их образования. В книге также обсуждаются технологии формирования и разрушения гидратов, раскрывается тема газогидратных залежей, найденных в природе, и их разработка и эксплуатация.

Таким образом, несмотря на большой фактический материал $[1,2,4,7-14]$, имеющийся в различных организациях, верхняя часть разреза глубокопогруженных отложений Южного Каспия с целью поисков газогидратов является наименее изученной и описанной в литературе зоной. Причем изучение зон газогидратов по сейсмическим данным произведено только на одной структуре Абшерон.

\section{Фактический материал и методика исследований}

При изучении верхней части разреза (ВЧР) глубокопогруженных отложений Южного Каспия использованы 17 региональных сейсмических профилей (рис. 1) и данные термодинамики региона [15]. По двум площадям Южного Каспия использованы данные скоростного анализа [13].

Газогидраты очень неустойчивое вещество, существующее в узком диапазоне температур и давления. Они встречаются на дне океанов (на глубинах около 500 м) и в полярных районах, где господствуют низкие температуры [16-18]. Оценивая диаграммы и графики зависимости термодинамического равновесия газогидратов, опубликованные разными авторами [18-20], можно прийти к выводу, что верхний предел формирования газогидратов располагается в интервале от 24 до 27 градусов для разных типов газа. Для метана максимум со- ставляет 24 градуса. Эти температуры [15] охватывают интервал до 500 м $[17,18]$. В связи с этим основная зона для поисков газогидратов ограничена результатами термодинамического моделирования (рис. 3, РТ). Перевод сейсмического материала из временного разреза в глубинный осуществлялся с помощью глубинно-временной диаграммы [13]. Расположения сейсмических профилей представлены на рис. 1. Интерпретация данных производилась с помощью лицензионного программного комплекса Kingdom Suite 2017. Применение сейсмических методов при изучении газогидратов основано на наличии прямой связи между особенностями геологического строения зон газогидратов и распределением в среде физических параметров. Индикатором газогидрата на временном разрезе могут быть аномальные изменения сейсмических характеристик волн: амплитуда, частота, фаза, скорости продольных и поперечных волн и т. д. Наиболее широко применяемым сейсморазведочным признаком газогидрата является наличие на сейсмических записях отражающего сейсмического горизонта BSR [18, 20, 21], а также ярких пятен $[18,20-22]$. По этим критериям интерпретированы все временные разрезы, указанные на рис. 1, и установлены верхняя и нижняя границы зон с отражениями, характерными для зон газогидратов.

\section{Результаты интерпретации}

На сейсмических профилях ВЧР характеризуется сложным волновым полем. Здесь выявляются разломы, грязевые вулканы, зоны с высоким содержанием газа, зоны газогидратов. При анализе сейсмических профилей (рис. 1) можно проследить аналогичные аномалии, выделенные при изучении зон газогидратов на площади Апшерон [5] и в разных регионах мира [20-37]. Ниже приведем описание сейсмических временных разрезов.

На рис. 2 (VII, 1) приведен фрагмент сейсмического временного разреза. Здесь морское дно прослеживается на времени от 0,4 до 0,5 с. Происходит последовательное погружение дна моря. В интервале от 0,6 до 0,75 с выделяются участки с характерными аномалиями, соответствующими 30нам газогидратов. По разрезу в интервале от 0,7 до 1,0 с прослеживаются разломы разного направления и порядка. Ниже этих зон по данным анализа амплитуды записи и теоретических основ устанавливается зона со свободным газом. На рис. 2 (VII, 2) представлен участок зоны газогидратов. Как видно, в нижней части профиля четко выделяются зоны газогидратов, а также зоны разломов. В ряде случаев по обе стороны от разлома наблюдаются разные углы наклона осей синфазности.

На рис. 2 показано продолжение временного разреза по профилю VII, фрагмент 3. Дно моря фиксируется в интервале времени от 0,97 до 1,1 с. Зона газогидратов прослеживается в интервале 1,15 до 1,35 с. В нижней части разреза выделяется серия разломов. 
Большой интерес представляет фрагмент временного разреза по профилю VIII, 4 (рис. 2). Стрелкой обозначено место отбора керна, содержащего газогидраты. По профилю установлено несколько протяженных горизонтов с интенсивной формой записи, соответствующих зонам газогидратов. Ниже этих зон не обнаружено присутствие разломов, выделенных ранее на других участках профиля VII, 2, 3. Однако в этих зонах выделяются аномалии, характерные для газа. В левой части профиля наблюдается прерывание осей синфазности горизонтов и некоторое изменение в форме записей.

На профиле I-I (рис. 3) выделяется соответствующая газогидратам граница и характерные аномалии. В зоне структур, а также в зонах расположения грязевых вулканов эти границы выделяются менее четко. Газогидраты установлены не по всей площади, а имеют определённые зоны распространения. Здесь установлены значительные глубины моря от 500 до $800 \mathrm{~m}$.

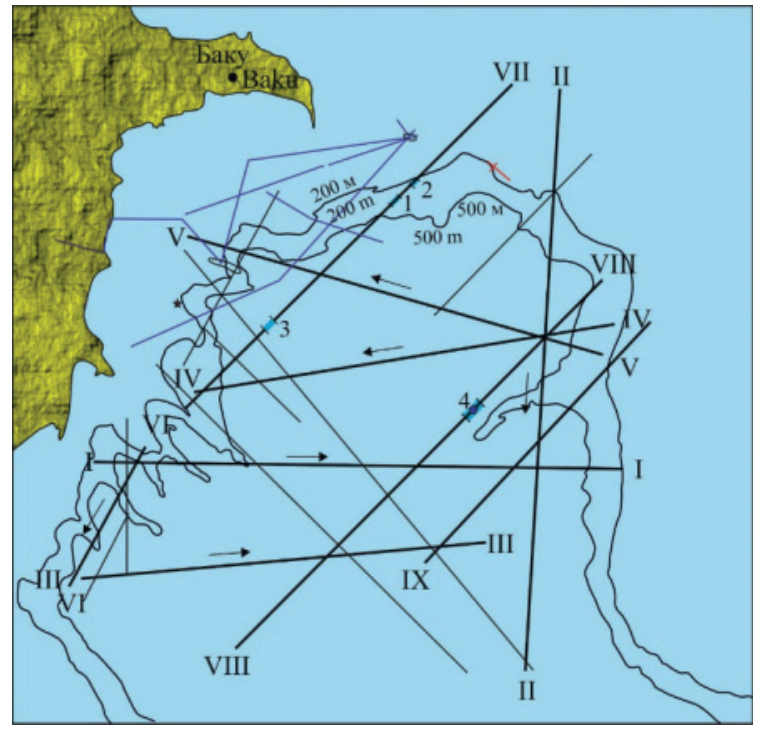

Рис. 1. Расположение сейсмических профилей в глубоководной зоне Южного Каспия

Fig. 1. Location of seismic profiles in the deep-sea zone of the South Caspian

На профиле V-V (рис. 3) также прослеживаются аналогичные зоны. Однако здесь интервал интенсивных аномалий, соответствующих границам BSR, располагается в интервале глубин от 0,8 до 1,6 с. Граница раздела среды морская вода-порода создает границу кратных отраженных волн, зарегистрированную на двойном времени пробега. В связи с этим на временном разрезе наблюдаются волны как от границ зон газогидратов, так и от границы кратно-отраженных волн, образованных от границы морского дна.

На профиле IX-IX (рис. 1) граница газогидратов маркируется в интервале времени от 0,4 до 0,9 с. В верхней части разреза ниже зон газогидратов наблюдаются разрывные нарушения и характерные газовые аномалии. Отражения от зон газо- гидратов выдержанные, четкие и при переходе в приподнятые участки прослеживаются фрагментарно.

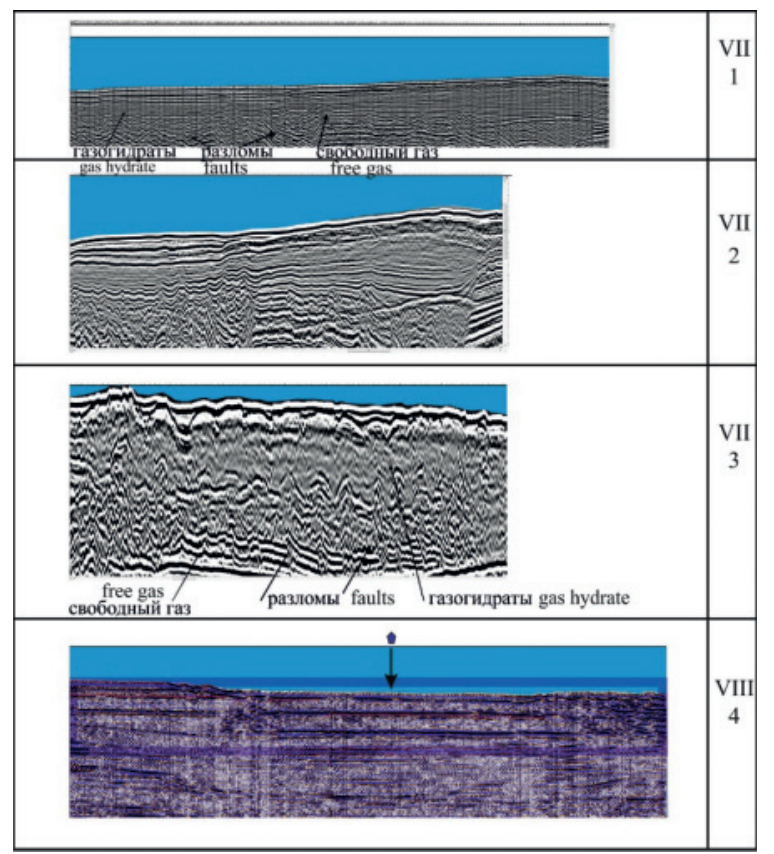

Рис. 2. Фрагменты временных сейсмических разрезов по профилям VII и VIII

Fig. 2. Fragments of temporary seismic sections along profiles VII and VIII

Указанные зоны маркированы на разных интервалах профиля III-III (рис. 3). Контрастность отражающих границ, расположенных на разрезе выше горизонта с газогидратами, меньше, чем расположенных под ним, то есть наблюдается некоторое «осветление» разреза. «Осветление» рассматривается как следствие уменьшения акустической контрастности отложений за счет присутствия гидратов в них. Далее фрагментарно установлены горизонты, форма записи которых указывает на их соответствие зонам газогидратов. На этом профиле структуры осложнены многочисленными разрывными нарушениями, наблюдаются аномалии, связанные с грязевулканической деятельностью, наличием газа.

Выделение границ газогидратов по профилям (I-IX) и дальнейшая их обработка в программном комплексе Kingdom Software 2017 позволила построить карты залегания границ газогидратов по площади. На рис. 4, 5 приведены глубины залегания кровли и подошвы зон газогидратов. Путем вычитания значений первой карты из второй получены мощности отложений (рис. 6). В центральной и юго-западной частях карты выделяются несколько зон с высокими мощностями отложений. В этих частях карты мощности отложений доходят более чем до 400 м, на фоне более пониженных участков от 180 до 260 м. Максимальные значения мощностей наблюдаются на структурах Д-13, Д-12 и между структурами Д-10 и Д-12. По сейс- 
мическим данным в этой зоне по кровле продуктивной толщи установлены разломы. Максимум также отмечен и в зоне структуры Д-44. Разломы установлены и на других структурах, однако мощности слоя здесь незначительные - от 100 до 260 м. Расчет объемов УВ газов газогидратов осуществлен для всей глубоководной части Южного Каспия, ограниченной изобатой 200 м.

\section{Оценка объемов углеводородных газов}

В результате интерпретации сейсмических временных разрезов, секущих глубоководную зону, выделены границы с интенсивными формами записи, указывавшие на границы аномальной зоны. Следует отметить, что степень заполнения порового пространства газогидратами по профилю неодинаковая, и этим обуславливается изменчивость в форме записи и интенсивности проявления газогидратов на профилях.

C целью оценки объемов газов в зонах газогидратов (GH) использована общепринятая формула:

$$
\mathrm{GH}=V \times \varnothing \times S_{h} \times F V,
$$

где $V$ - объем породы, определенный по данным сейсмических методов исследований; $\varnothing-$ средняя пористость в зоне газогидратов; $S_{h}$ - степень емкостного заполнения пористости газогидратов, $F V$ коэффициент расширения метана при разложении газогидрата (обычно принят как 160). Вводные и расчётные данные приведены в табл. 1 .

Таблица 1. Расчеты объемов УВ газов в зонах газогидратов

Table 1. Calculations of hydrocarbon gas volumes within gas hydrate zones

\begin{tabular}{|c|c|c|c|c|c|}
\hline 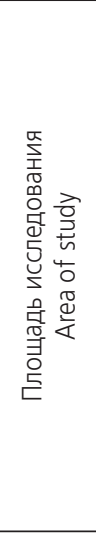 & 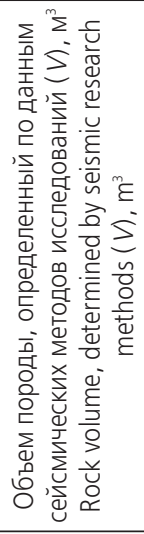 &  &  & 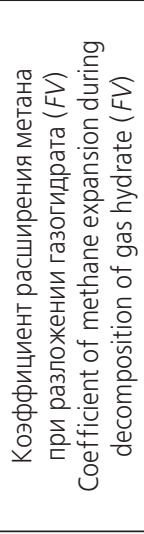 & 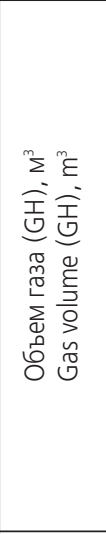 \\
\hline $\begin{array}{l}\text { Общая } \\
\text { General }\end{array}$ & $6,99365 \cdot 10^{12}$ & 0,20 & 0,036 & 160 & $8,00 \cdot 10=$ \\
\hline
\end{tabular}

Следует отметить, что средняя величина пористости в зоне газогидратов была занижена на $50 \%$, что позволит более обосновано подойти к изучению объемов УВ газа газогидратов. $S_{h}$-значения выбраны и использованы исходя из анализа результатов, полученных на двух скоплениях газогидратов Боздаг и Элм, а также из анализа данных $[1,2,4,38]$. Значение $S_{h}$ для глин составляет 0,036. Расчеты произведены для всей глубоководной зоны, объем пород которой составляет $6,99365 \cdot 10^{12}$. Как видно из приведенных расчетов суммарные объемы газа, рассчитанные для изучаемой площади отложений,

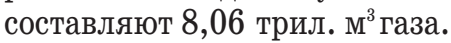

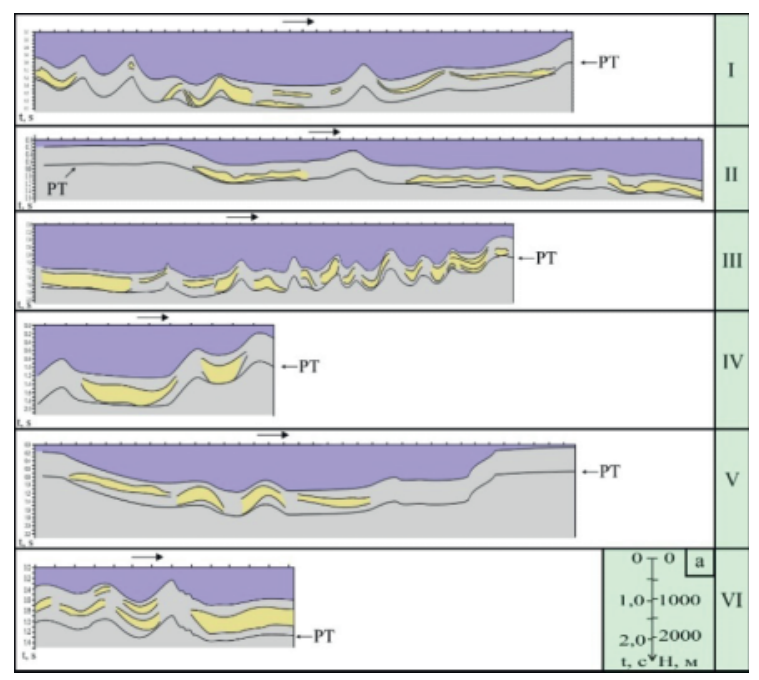

Рис. 3. Результаты интерпретации сейсмических данных с выделением зон газогидратов (желтый фон), VI a скоростная кривая (приблизительно)

Fig. 3. Results of interpretation of seismic data with the identification of gas hydrate zones (yellow background), VI a - TD conversion (approximately)

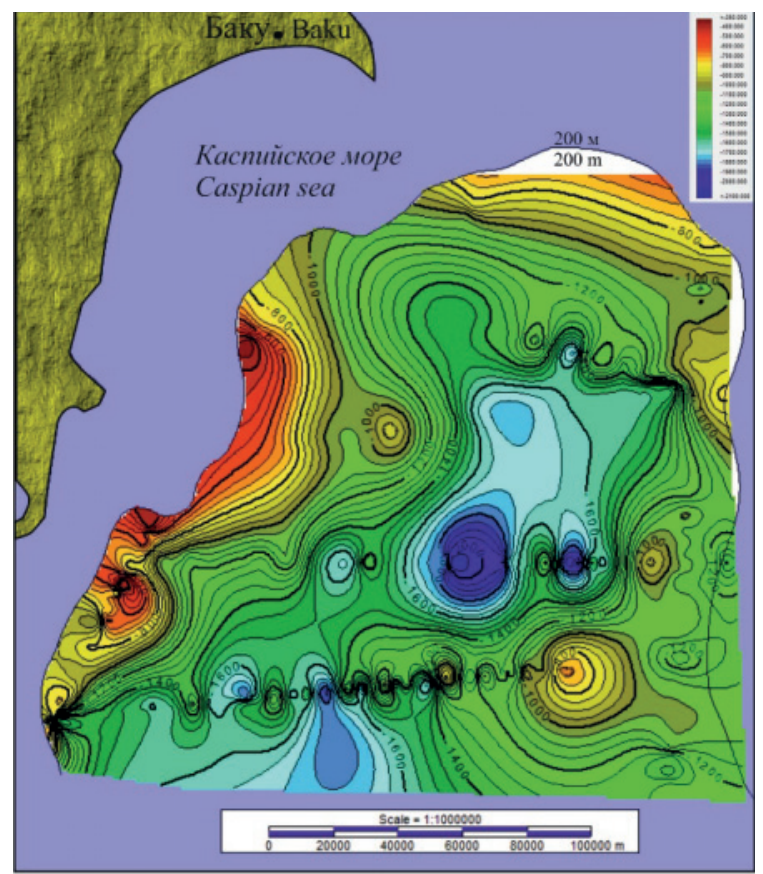

Рис. 4. Карта верхней границы зоны газогидратов в пределах Южного Каспия

Fig. 4. Map of the upper boundary of the gas hydrate zone within the South Caspian

Также рассчитан объем газогидратов, содержащихся в породе в пределах структур. По карте нефтяных залежей и структур, составленной Государственной Нефтяной Компанией Азербайджанской Республики [8], рассчитаны размеры всех глубокопогруженных структур глубоководной части Юж- 
ного Каспия. Объем пород рассчитывался по данным мощностей газогидратов, выявленных в зонах расположения структур (рис. 7), установленных по сейсмическим данным. Результаты расчетов занесены в табл. 2. Расчеты произведены для всей глубоководной зоны, объем пород которой составляет $6,99365 \cdot 10^{12}$ в в то время как общий объем пород в зоне расположения структур $1,25159 \cdot 10^{12}$. Ранжирование данных по объемам в пределах структур (рис. 7) показало, что в 10 первых структурах: 1) Д-11; 2) Д-60; 3) Д-10; 4) Д-28; 5) Д-25; 6) Д-16; 7) Д-41; 8) Д-19; 9) Д-13; 10) Д-71 содержится $6,12 \cdot 10^{11} \mathrm{M}^{3}$ газа, в то время как для всех остальных 46 структур объем составляет $6,40 \cdot 10^{11} \mathrm{~m}^{3}$ газа. Исходя из сопоставления различных данных установлено, что многие из этих структур осложнены разломами, выделенными по кровле продуктивной толщи.

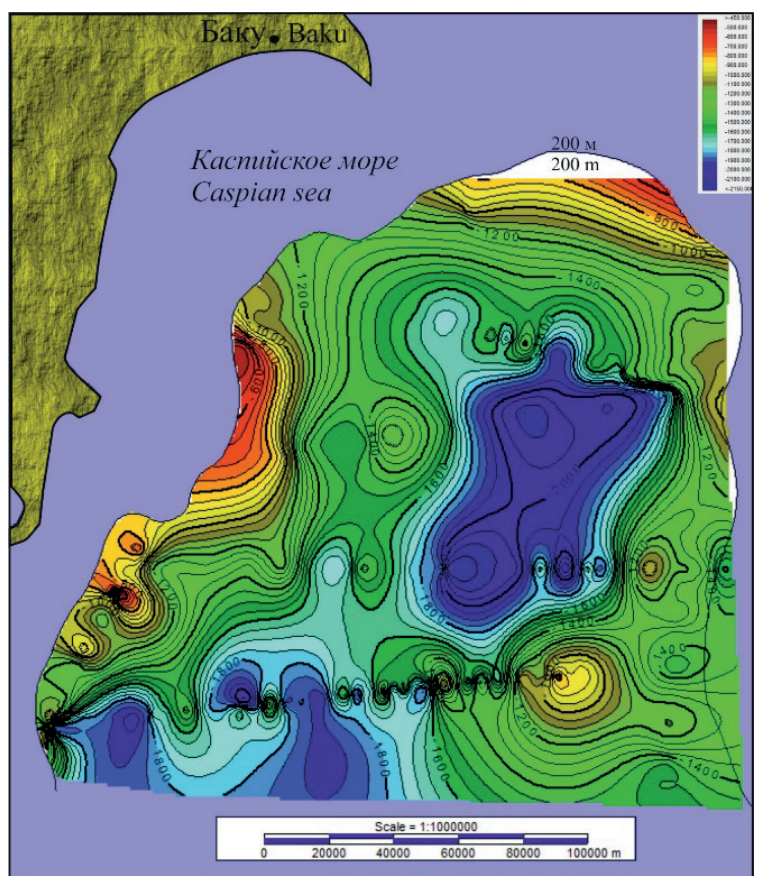

Рис. 5. Карта нижней границы зоны газогидратов в пределах Южного Каспия

Fig. 5. Map of the lower boundary of the gas hydrate zone within the South Caspian

Исходя из вышеизложенного в глубоководной части Южного Каспия выделяются две перспективно-газогидратные зоны:

- высокоперспективная зона в южной части (I);

- перспективная зона в северной глубоководной части (II).

Перечисленные зоны накопления газогидратов обеспечат энергетическую безопасность Азербайджана на многие годы.

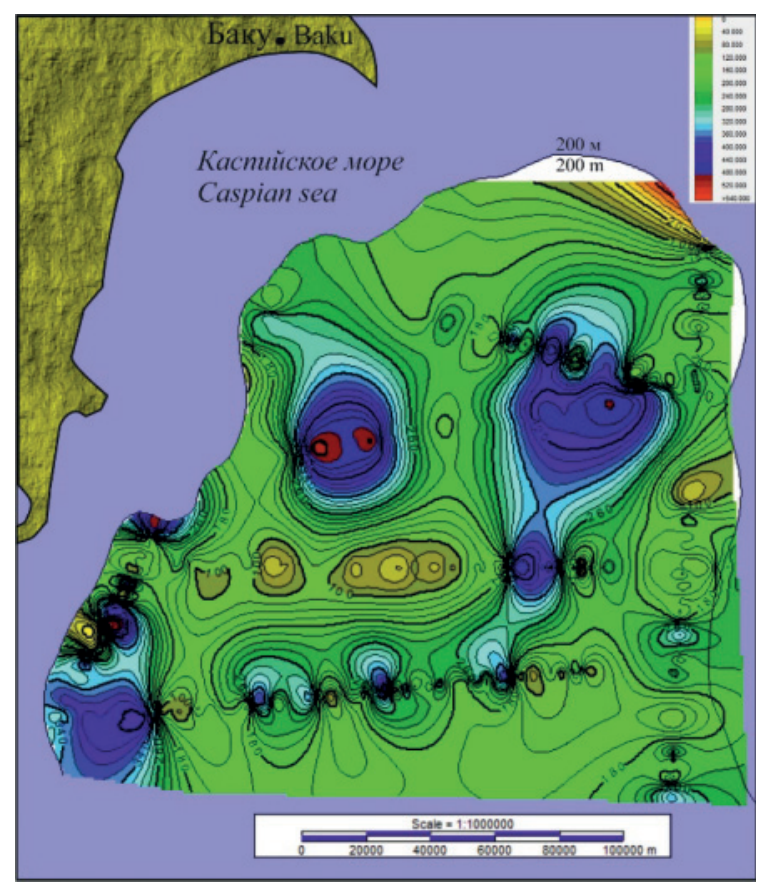

Рис. 6. Карта мощностей зоны газогидратов в пределах Южного Каспия

Fig. 6. Map of thicknesses of gas hydrate zone within the South Caspian

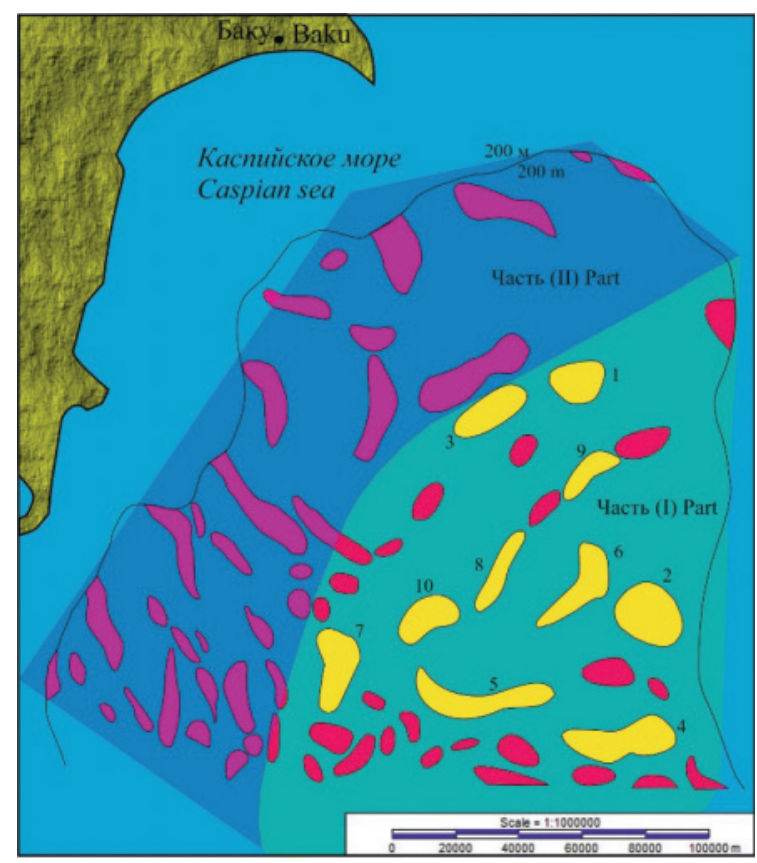

Рис. 7. Карта распределения объемов газа по площади

Fig. 7. Map of gas volumes distribution by area 
Таблица 2. Расчет объемов УВ газов в пределах нефтяных и газовых месторождений

Table 2. Calculations of hydrocarbon gas volumes within oil and gas fields

\begin{tabular}{|c|c|c|}
\hline $\begin{array}{l}\text { Месторождения } \\
\text { Oil and gas fields }\end{array}$ & $\begin{array}{c}\text { Объем породы, } \\
\text { Rock volume (V), } \\
10^{6} \mathrm{~m}^{3}\end{array}$ & $\begin{array}{c}\mathrm{GH}=V \times \varnothing \times S_{h} \times F V \\
\varnothing=0,20, S_{h}=0,0036 \\
F V=160,10^{6} \mathrm{~m}^{3}\end{array}$ \\
\hline $\mathrm{D}-2$ & 32958,4405 & 37968,12346 \\
\hline $\mathrm{D}-10$ & 60807,7944 & 70050,57915 \\
\hline D-1 & 27785,1855 & 32008,5337 \\
\hline D-4 & 7978,9601 & 9191,762035 \\
\hline D-6 & 21559,7154 & 24836,79214 \\
\hline D-7 & 6358,1219 & 7324,556429 \\
\hline D-11 & 75759,1614 & 87274,55393 \\
\hline$D-12$ & 33115,5981 & 38149,16901 \\
\hline $\mathrm{D}-13$ & 41041,4154 & 47279,71054 \\
\hline D-14 & 22273,9195 & 25659,55526 \\
\hline $\mathrm{D}-15$ & 19311,2966 & 22246,61368 \\
\hline $\mathrm{D}-16$ & 48512,3787 & 55886,26026 \\
\hline $\mathrm{D}-17$ & 5566,6143 & 6412,739674 \\
\hline $\mathrm{D}-18$ & 11635,6220 & 13404,23654 \\
\hline D-19 & 41819,3852 & 48175,93175 \\
\hline $\mathrm{D}-20$ & 7760,4542 & 8940,043238 \\
\hline $\mathrm{D}-21$ & 26504,8346 & 30533,56946 \\
\hline$D-23$ & 20437,0597 & 23543,49277 \\
\hline$D-24$ & 6636,4740 & 7645,218048 \\
\hline$D-25$ & 49283,2461 & 56774,29951 \\
\hline$D-26$ & 5446,3951 & 6274,247155 \\
\hline $\mathrm{D}-27$ & 5016,3230 & 5778,804096 \\
\hline$D-28$ & 55471,8664 & 63903,59009 \\
\hline$D-28$ & 13173,7810 & 15176,19571 \\
\hline D-29 & 11913,2817 & 13724,10052 \\
\hline$D-35$ & 35471,2944 & 40862,93115 \\
\hline D-37 & 8713,2820 & 10037,70086 \\
\hline D-39 & 12626,9149 & 14546,20596 \\
\hline D-40 & 7955,3805 & 9164,598336 \\
\hline D-41 & 48025,2394 & 55325,07579 \\
\hline D-42 & 13417,0433 & 15456,43388 \\
\hline$D-43$ & 7201,4820 & 8296,107264 \\
\hline D-44 & 15755,2266 & 18150,02104 \\
\hline D-45 & 5450,9067 & 6279,444518 \\
\hline D-46 & 4603,0470 & 5302,710144 \\
\hline $\mathrm{D}-47$ & 3252,1998 & 3746,53417 \\
\hline D-50 & 11728,8915 & 13511,68301 \\
\hline D-52 & 6126,5750 & 7057,8144 \\
\hline D-53 & 2084,4478 & 2401,283866 \\
\hline D-55 & 6747,4352 & 7773,04535 \\
\hline D-56 & 8997,5932 & 10365,22737 \\
\hline D-58 & 8253,3560 & 9507,866112 \\
\hline D-59 & 13593,2846 & 15659,46386 \\
\hline D-60 & 71139,4168 & 81952,60815 \\
\hline D-61 & 10627,9879 & 12243,44206 \\
\hline D-62 & 17334,8249 & 19969,71828 \\
\hline D-64 & 11846,5955 & 13647,27802 \\
\hline D-65 & 2641,5269 & 3043,038989 \\
\hline D-66 & 1691,9655 & 1949,144256 \\
\hline D-67 & 4998,2340 & 5757,965568 \\
\hline D-68 & 3793,5570 & 4370,177664 \\
\hline D-69 & 5097,3253 & 5872,118746 \\
\hline D-71 & 38960,1687 & 44882,11434 \\
\hline$D-72$ & 5726,2312 & 6596,618342 \\
\hline D-74 & 12360,4016 & 14239,18264 \\
\hline$D-38$ & 22100,8823 & 25460,21641 \\
\hline
\end{tabular}

\section{Выводы}

Несмотря на большой фактический материал, имеющийся в различных организациях, верхняя часть разреза глубокопогруженных отложений Южного Каспия с точки зрения поисков газогидратов является наименее изученной и описанной в литературе зоной. Анализ опубликованных и неопубликованных материалов по газогидратам Каспийского моря показал, что в основном все работы базируются лишь на изучении скопления газогидратов Элм, Боздаг, площади Абшерон, а также термодинамических факторах региона с целью определения возможной зоны их скопления. Следует отметить, что по сейсмическим данным проведены исследования только для структуры Абшерон.

При изучении газогидратов глубокопогруженных отложений Южного Каспия использованы 17 региональных сейсмических профилей и данные термодинамики региона. По двум площадям Южного Каспия использованы данные скоростного анализа.

Анализ термодинамических данных показал, что в пределах Южного Каспия газогидраты могут быть установлены в интервале 0,2-2,2 с, выбранном для последующей интерпретации. Интерпретация данных производилась с помощью лицензионного программного комплекса Kingdom Suite 2017 и по признакам выделения зон газогидратов на сейсмических записях отражающего сейсмического горизонта BSR, а также ярких пятен. Перевод сейсмического материала из временного разреза в глубинный осуществлялся с помощью глубинно-временной диаграммы.

В результате интерпретации сейсмических временных разрезов, секущих глубоководную зону, выделены границы с интенсивными формами записи, указывающими на аномальность этих границ. Следует отметить, что степень заполнения порового пространства газогидратами по профилю различная, и этим обуславливается изменчивость в форме записи и интенсивности проявления газогидратов на профилях.

Расчеты произведены для всей глубоководной зоны, объем пород которой составляет $6,99365 \cdot 10^{12}$. Суммарные объемы газа, рассчитанные для изучаемой площади, составляют 8,06 трил м газа. Также рассчитан объем газогидратов, содержащихся в породе в пределах структур. Ранжирование данных по объемам показало, что в южной части Каспия содержится максимальное количество газа.

Исходя из вышеизложенного в глубоководной части Южного Каспия выделяются две перспективно-газогидратные зоны: высокоперспективная зона в южной части; перспективная зона в северной глубоководной части.

Перечисленные зоны накопления газогидратов обеспечат энергетическую безопасность Азербайджана на многие годы. 


\section{СПИСОК ЛИТЕРАТУРЫ}

1. Гусейнов Р.А., Дадашев Ф.Г. Углеводородные газы Каспийского моря. - Баку: Нафта-Пресса, 2000. - 128 с.

2. Подводногрязевулканический тип скоплений газовых гидратов / Г.Д. Гинсбург, И.С. Грамберг, И.С. Гулиев, Р.А. Гусейнов, А.А. Дадашев, В.Л. Иванов, А.Г. Кротов, Ч.С. Мурадов, В.А. Соловьев, Е.В. Телепнев // Доклады Академии Наук CCCP. - 1988. - T. 300. - № 2. - C. 416-418.

3. Muradov Ch.S. The Area of formation of the South Caspian gas hydrates // South-Caspianbasin: geology, geophysics, oil and gas content. - Baku: Nafta-Press,2004. - P. 322-332.

4. Гинсбург Г.Д., Соловьев В.А. Субмаринные газовые гидраты. СПб.: ВНИИОкеангеология, 1994. - 193 с.

5. Diaconescu C.C., Kieckhefer R.M., Knapp J.H. Geophysical evidence for gas hydrates in the deep water of the South Caspian Basin Azerbaijan // Marine and Petroleum Geology. - 2001. V. 18. - P. 209-221.

6. Панахов Р.А., Адуллаев Э.А., Новрузова С.Г. Гозовые гидраты. - Баку: Элм, 2012. - 298 с.

7. Xəzər dənizinin Abşeron arxipelağı və Bakı arxipelağının şimal hissəsində Azərbaycan sektorunda kəsilişin üst hissəsinin qazllıı̆ı və dəniz dibi qaz çıxıslarının (qaz-hidratlar) öyrənilməsi məqsədilə geoloji-geofiziki və qazma məlumatlarının ümumiləşdirilməsi. Bakı: ARDNŞ, 2012. - $215 \mathrm{~s}$.

8. Геология Азербайджана. Нефть и газ / под ред. Ак.А. Али-заде. - Баку: Изд-во «Nafta-Press», 2008. - T. VII. - 380 c.

9. Геология Азербайджана. Гидрогеология и инженерная геология / под ред. Ак.А. Али-заде. - Баку: Изд-во «Nafta-Press», 2008. - T. VIII. - 380 c.

10. Гулиев И.С., Дадашев Ф.Г. Полетаев А.В. Изотопы углеводородных газов Азербайджана. - Баку: Изд-во «Nafta-Press», 2013. - $107 \mathrm{c}$.

11. Методология изучения и освоения месторождения Булла-дениз / под ред. Ак.А. Али-заде. - Баку: Национальная Академии Наук Азербайджана, Институт глубинных проблем, 1995. - $138 \mathrm{c}$.

12. Guliyev I.S., Levin L.E., Fedorov D.L. Hydrocarbons potential of the Caspian region (System Analysis). - Baku: Nafta-Press, 2003. $-120 \mathrm{p}$.

13. South Caspian Basin project. Geological Institute of Azerbaijan National Academy of Sciences (GIA), Azerbaijan research Institute of Geophysics (SOCAR), Earth Sciences and Resources Institute of the South Carolina (ESRI). - 1995. - $171 \mathrm{p}$.

14. South-Caspian Basin: geology, geophysics, oil and gas content / ed. by A.A. Ali-Zadeh. - Baku: Nafta-Press, 2004. - 333 p.

15. Geothermal Atlas of Azerbaijan. Geological Institute of Azerbaijan National Academy of Sciences / Eds. A.A. Ali-Zadeh, S.A. Aliyev. $-2001 .-151$ p.

16. Дмитриевский А.Н., Баланюк И.Е. Газогидраты морей и океанов - источник углеводородов будущего. - М.: 000 ИРЦ Газпром, 2009. -415 с.

17. Макогон Ю.Ф. Газогидраты. История изучения и перспективы освоения // Геология и полезные ископаемые Мирового океана. - 2010. - № 2. - P. 1-17.

18. Naresh Kumar Thakur, Sanjeev Rajput. Exploration of Gas Hydrates Geophysical Techniques. - Berlin, Heidelberg: SpringerVerlag,2011. - $281 \mathrm{p}$.

19. Полетаев А.В., Полетаева Е.В. Изученность диаграмм гетерогенного равновесия в координатах давления и температуры: гидратообразования // Перспективы развития научного знания в XXI веке. Экономика России: проблемы, тенденции, перспективы. -Тамбов: ИД ТГУ,2015. - Вып. 4. - С. 104-111.

20. Sloan D.E., Koh C.A. Clathrate Hydrates of Natural Gases. $3^{\text {rd }}$ ed. - Boca Raton: CRC Press, 2007. - 721 p.

21. Bangs N.L.B., Hornbach M.J., Berndt Ch. The mechanics of intermittent methane venting at South Hydrate Ridge inferred from 4D seismic surveying // Earth and Planetary Science Letters. - 2011. - V. 310. - P. 105-112.

22. Kalachand Sain, Harsh Gupta. Gas hydrates in India: Potential and development. - India: Gondwana Research, 2012. P. 112-131.

23. Hamidreza Yarveicy, Mohammad M. Ghiasi, Amir H. Mohammadi. Determination of the gas hydrate formation limits to isenthalpic Joule-Thomson expansions / Chemical Engineering Research and Design. - 2018. - V. 132. -P. 208-214.

24. Geophysical characterization of a fine-grained gas hydrate reservoir in the Shenhu area, northern South China Sea: Integration of seismic data and downhole logs / Jiliang Wang, Shiguo Wu, Xiu Kong, Qingping Li, Jianxin Wang, Rong Ding // Marine and Petroleum Geology. - 2018. - V. 92. - P. 895-903.

25. Musakaev N.G., Khasanov M.K., Borodin S.L., The mathematical model of the gas hydrate deposit development in permafrost // International Journal of Heat and Mass Transfer. - 2018. V. 118. - P. $455-461$.

26. Şükrü Merey, Sotirios Nik Longinos. Numerical simulations of gas production from Class 1 hydrate and Class 3 hydrate in the Nile Delta of the Mediterranean Sea // Journal of Natural Gas Science and Engineering. - 2018. - V. 52. - P. 248-266.

27. Lorenson Th.D., Collett T.S. National Gas Hydrate Program Expedition 01 offshore India; gas hydrate systems as revealed by hydrocarbon gas geochemistry // Marine and Petroleum Geology. 2018. - V. 92. - P. 477-492.

28. Estimation of seismic velocities and gas hydrate concentrations: a case study from the Shenhu area, northern South China Sea/ Jie Liu, Jianzhong Zhang, Fei Ma, Ming Wang, Yunbao Sun // Marine and Petroleum Geology. - 2017. - V. 88. - P. 225-234.

29. High-resolution seismic characterization of the gas and gas hydrate system at Green Canyon 955, Gulf of Mexico, USA / S.S. Haines, P.E. Hart, T.S. Collett, W. Shedd, M. Frye, P. Weimer, R. Boswell // Marine and Petroleum Geology. - 2017. - V. 82. P. 220-237.

30. Zijian Zhang, Wright Ch.S. Quantitative interpretations and assessments of a fractured gas hydrate reservoir using three-dimensional seismic and LWD data in Kutei basin, East Kalimantan, offshore Indonesia // Marine and Petroleum Geology. - 2017. V. 84. - P. 257-273.

31. Musakaev N.G., Khasanov M.K., Borodin S.L. The mathematical model of the gas hydrate deposit development in permafrost // International Journal of Heat and Mass Transfer. - 2018. V. 118 . - P. $455-461$.

32. Differential fluid migration behaviour and tectonic movement in Lower Silurian and Lower Cambrian shale gas systems in China using isotope geochemistry / Hao Xu, Wen Zhou, Qian Cao, Christopher Xiao, Qiumei Zhou, Haotian Zhang, Yeyu Zhang // Mar. Pet. Geol. - 2017. - V. 89. - P. 47-57.

33. Seismic evidence and formation mechanism of gas hydrates in the Zhongjiannan Basin, Western margin of the South China Sea / Yintao Lu, Xiwu Luan, Fuliang Lyu, Bin Wang, Zhili Yang, Taotao Yang, Genshun Yao // Marine and Petroleum Geology. 2017. - V. 84. - P. 274-288.

34. Genetic types of gas hydrates in China/ Jinxing Dai, Yunyan Ni, Shipeng Huang, Weilong Peng, Wenxue Han, Deyu Gong, Wei Wei // Petroleum Exploration and Development. - 2017. V. $44 .-$ P. 887-898.

35. Hydrocarbon source for oil and gas indication associated with gas hydrate and its significance in the Qilian Mountain permafrost, Qinghai, Northwest China / Bin Cheng, Jianbing Xu, Zhenquan $\mathrm{Lu}$, Yonghong Li, Weichao Wang, Shan Yang, Hu Liu, Ting Wang, Zewen Liao // Mar. Pet. Geol. - 2017. - V. 89. P. 202-215.

36. Methane source linked to gas hydrate system at hydrate drilling areas of the South China Sea: ore water geochemistry and numerical model constraints/ Yu Hu, Min Luo, Linying Chen, Qiany- 
ong Liang, Dong Feng, Jun Tao, Shengxiong Yang, Duofu Chen // Journal of Asian Earth Sciences. - 2018. - V. 44. P. 2-15.

37. Contribution of thermogenic organic matter to the formation of biogenic gas hydrate: Evidence from geochemical and microbial characteristics of hydrate-containing sediments in the Taixinan Basin, South China Sea / Junli Gong, Xiaoming Sun, Li Xu,
Hongfeng Lu // Marine and Petroleum Geology. - 2017. V. 80 . - P. 432-449.

38. Frye M., Shedd W., Boswell R. Gas hydrate resource potential in the Terrebonne Basin, Northern Gulf of Mexico // Marine and Petroleum geology. - 2011. - V. 34. - P. 1-19.

Поступила 18.09.2018 г.

\section{Информация об авторах}

Полетаев A.B., кандидат геолого-минералогических наук, ведущий научный сотрудник Института нефти и газа Национальной Академии Наук Азербайджана.

Полетаева $\boldsymbol{E} . \boldsymbol{B} .$, доктор философии по геологии и минералогии, ведущий научный сотрудник Института нефти и газа Национальной Академии Наук Азербайджана. 
UDK $550.3,550.31$

\title{
EVALUATION OF VOLUME OF GAS HYDRATE HYDROCARBON GASES WITHIN AZERBAIJAN SECTOR OF SOUTHERN CASPIAN ON SEISMIC DATA
}

\author{
Alexander V. Poletaev', \\ avo1@mail.ru \\ Elena V. Poletaeva', \\ yelenapv@mail.ru \\ 1 National Academy of Sciences of Azerbaijan, \\ 9, F. Amirov street, Baku, AZ1000, Azerbaijan.
}

The relevance of the research is in studying the upper part of the section of Southern Caspian deep part to distinguish gas hydrate zones by seismic data, to evaluate their thickness as well as calculation of gas hydrate gas volumes. The study of gas hydrates in the South Caspian is necessary for reliable assessment of energy potential of hydrocarbon gases in Azerbaijan. The results of the study of gas hydrates can also be applied to assess the role of gas hydrates in the near-surface layers of the geosphere, especially in connection with their possible impact on ecology of the Caspian Sea, the region and global climate change.

The aim of the research is to identify and estimate the volumes of hydrocarbon gases of gas hydrates of the deep-water part of the South Caspian by seismic data.

The object of the research - 17 regional seismic profiles and data on thermodynamics of the region. When interpreting seismic profiles the authors have emphasized the identification of horizons containing gas hydrates.

Research methods are based on the presence of a direct relationship between the features of the geological structure of the gas hydrate zones and distribution of physical parameters in the medium. Anomalous changes in the seismic characteristics of waves can serve as indicators of gas hydrate at a time section: amplitude, frequency, phase, velocity of longitudinal and transverse waves. The presence of the reflecting seismic horizon BSR, as well as bright spots on the seismic records is widely used seismic attribute of gas hydrate. All the time sections are interpreted according to these criteria and the upper and lower boundaries of the zones with reflections characteristic of the gas hydrate zones are established. The data were interpreted using the licensed software package Kingdom Suite 2017.

As a result of the research, the authors constructed the maps of the lower and upper boundaries of the gas hydrate zones, and calculated the thickness of the deposits containing gas hydrates. The rock volumes determined by seismic data enabled to calculate the volumes of hydrocarbon gases contained in gas hydrates. The maximum volume of gas in the investigated zone is $8,06 \cdot 10^{12}$ of gas. The volume of gas hydrates contained in the rock within the structures was calculated as well. Ranking of the data by volume showed that the southern part of the Caspian contains the maximum amount of gas. Proceeding from the above, two perspective-gas hydrate zones can be distinguished in the deep-water part of the South Caspian: highly prospective zone in the southern part; prospecting zone in the northern deep-water part. These gas hydrate accumulation zones will ensure the energy security of Azerbaijan for many years.

Key words:

Gas hydrates, seismic profiles, estimation, gas, South Caspian, hydrocarbon.

\section{REFERENCES}

1. Guseynov R.A., Dadashev F.G. Uglevodorodnye gazy kaspiyskogo morya [Hydrocarbon gases of the Caspian Sea]. Baku, Nafta-Pres$\mathrm{sa}, 2000.128 \mathrm{p}$.

2. Ginsburg G.D., Gramberg I.S., Guliev I.S., Guseynov R.A., Dadashev A.A., Ivanov V.L., Krotov A.G., Muradov Ch.S., Solovev V.A., Telepnev E.V. Podvodnogryazevulkanicheskiy tip skopleniy gazovykh gidratov [Subsea mud volcanoes type of the gas hydrate fields]. Doklady Akademii Nauk SSSR, 1988, vol. 300, no. 2, pp. 416-418.

3. Muradov Ch.S. The Area of formation of the South Caspian gas hydrates. South-Caspian basin: geology, geophysics, oil and gas content. Baku, Publishing House Nafta-Press, 2004. pp. $322-332$.

4. Ginsburg G.D., Solovev V.A. Submarinnye gazovye gidraty [Submarine gas hydrates]. St. Petersburg, VNII Okeangeologiya Publ., 1994.199 p.

5. Diaconescu C.C., Kieckhefer R.M., Knapp J.H. Geophysical evidence for gas hydrates in the deep water of the South Caspian Basin Azerbaijan. Marine and Petroleum Geology, 2001, vol. 18, pp. 209-221.

6. Panakhov R.A., Adullaev E.A., Novruzova S.G. Gazovye gidraty [Gas hydrates]. Baku, Elm Publ., 2012. 298 p.

7. Xəzər dənizinin Abşeron arxipelağı və Bakı arxipelağının şimal hissəsindəAzərbaycan sektorunda kəsilișin üst hissəsinin qazlilığı və dəniz dibi qaz çıxıslarının (qaz-hidratlar) öyrənilməsi məqsadila geoloji-geofiziki va qazma məlumatlarının ümumiləşdirilməsi [Gas hearing capacity in upper part of section and sea bottom gas shows (gas hydrates) in Absheron archipelago and northern part of Baku archipelago of Azerbaijan sector Caspian Sea with aims geological, geophysical and drilling data collection]. Baku, SOCAR Publ., 2012. 215 p.

8. Geologiya Azerbaydzhana [Geology of Azerbaijan]. Tom VII. Neftigaz. Vol. VII. Oil and gas]. Ed. by A.A. Ali-Zadeh. Baku, NaftaPress Publ. house, 2008. 380 p.

9. Geologiya Azerbaydzhana. Tom VIII. Gidrogeologiya i inzhenernaya geologiya [Hydrogeology and engineering geology. Vol. VIII. Hydrogeology and engineering geology]. Ed. by A.A. Ali-Zadeh. Baku, Nafta-Press, 2008. 380 p.

10. Guliev I.S., Dadashev F.G. Poletaev A.V. Izotopy uglevodorodnykh gazov Azerbaydzhana [Isotopes of hydrocarbon gases of Azerbaijan]. Baku, Nafta-Press, 2013. 107p.

11. Metodologiya izucheniy ai osvoeniya mestorozhdeniya Bulla-Deniz [Methodology of study and developing the Bulla-Deniz field, the National Academy of Sciences of Azerbaijan]. Ed. by A.A. AliZadeh. Baku, National Academy of Sciences of Azerbaijan, the Institute of Deep Problems, 1995. 138 p.

12. Guliyev I.S., Levin L.E., Fedorov D.L. Hydrocarbons potential of the Caspian region (System Analysis). Baku, Nafta-Press, 2003. 120. p. 
13. South Caspian Basin project. Geological Institute of Azerbaijan National Academy of Sciences (GIA), Azerbaijan research Institute of Geophysics (SOCAR), Earth Sciences and ResourcesInstitute of the South Carolina (ESRI). 1995. $171 \mathrm{p}$.

14. South-Caspian Basin: geology, geophysics, oil and gas content. Ed. by A.A. Ali-Zadeh. Baku, Nafta-Press, 2004. 333 p.

15. Geothermal Atlas of Azerbaijan. Geological Institute of Azerbaijan National Academy of Sciences. Eds. A.A. Ali-Zadeh, S.A. Aliyev. 2001.151 p.

16. Dmitrievskiy A.N., Balanyuk I.E. Gazogidraty Morey i Okeanov - Istochnik Uglevodorodov Budushchego [Gas hydrates of the sea and Ocean - the future hydrocarbon source]. Moscow, IRTs GAZPROM Publ., 2009. 416 p.

17. Makogon Yu.F. Gas hydrates. History of study and perspectives of development. Geology and mineral resources of the world ocean, 2010, no. 2, pp. 1-17. In Rus.

18. Naresh Kumar Thakur, Sanjeev Rajput. Exploration of Gas Hydrates Geophysical Techniques. Berlin, Heidelberg, Springer-Verlag, 2011. $281 \mathrm{p}$.

19. Poletaev A.V., Poletaeva E.V. Izuchennost diagram geterogennogo ravnovesiya v koordinatakh davleniya i temperatury: gidratoobrazovaniya [Study of heterogenous equilibrium in pressure and temperature coordinates: formation of hydrates]. Perspektivy razvitiya nauchnogo znaniya VXXI veke. Ekonomika Rossii: proble$m y$, tendentsii, perspektivy [Perspective of scientific knowledge development in the XXI century. Russian economic: problems, tendentious, perspectives]. Tambov, TSU Publ. house, 2015. Iss. 4, pp. 104-111.

20. Sloan D.E., Koh C.A. Clathrate Hydrates of Natural Gases. $3^{\text {rd }}$ ed. Boca Raton, CRC Press, 2007. 721 p.

21. Bangs N.L.B., Hornbach M.J., Berndt Ch. The mechanics of intermittent methane venting at South Hydrate Ridge inferred from 4D seismic surveying. Earth and Planetary Science Letters, 2011, vol. 310, pp. 105-112.

22. Kalachand Sain, Harsh Gupta. Gas hydrates in India: Potential and development. India, Gondwana Research, 2012. pp. 112-131.

23. Hamidreza Yarveicy, Mohammad M. Ghiasi, Amir H. Mohammadi. Determination of the gas hydrate formation limits to isenthal pic Joule-Thomson expansions. Chemical Engineering Research and Design, 2018, vol. 132, pp. 208-214.

24. Jiliang Wang, Shiguo Wu, Xiu Kong, Qingping Li, Jianxin Wang, Rong Ding. Geophysical characterization of a fine-grained gas hydrate reservoir in the Shenhu area, northern South China Sea: Integration of seismic data and down hole logs. Marine and Petroleum Geology, 2018, vol. 92, pp. 895-903.

25. Musakaev N.G., Khasanov M.K., Borodin S.L. The mathematical model of the gas hydrate deposit development in permafrost. In ternational Journal of Heat and Mass Transfer, 2018, vol. 118, pp. $455-461$

26. Şükrü Merey, Sotirios Nik Longinos. Numerical simulations of gas production from Class 1 hydrate and Class 3 hydrate in the Nile Delta of the Mediterranean Sea. Journal of Natural Gas Science and Engineering, 2018, vol. 52, pp. 248-266.

27. Lorenson Th.D., Collett T.S. National Gas Hydrate Program Expedition 01 offshore India; gas hydrate systems as revealed by hy- drocarbon gas geochemistry. Marine and Petroleum Geology, 2018, vol. 92, pp. 477-492.

28. Jie Liu, Jianzhong Zhang, Fei Ma, Ming Wang, Yunbao Sun. Estimation of seismic velocities and gas hydrate concentrations: a case study from the Shenhu area, northern South China Sea. $M a$ rine and Petroleum Geology, 2017, vol. 88, pp. 225-234.

29. Haines S.S., Hart P.E., Collett T.S., Shedd W., Frye M., Weimer P., Boswell R. High-resolution seismic characterization of the gas and gas hydrate system at Green Canyon 955, Gulf of Mexico, USA. Marine and Petroleum Geology, 2017, vol. 82, pp. 220-237.

30. Zijian Zhang, Wright Ch.S. Quantitative interpretations and assessments of a fractured gas hydrate reservoir using three-dimensional seismic and LWD data in Kutei basin, East Kalimantan, offshore Indonesia. Marine and Petroleum Geology, 2017, vol. 84 , pp. 257-273.

31. Musakaev N.G., Khasanov M.K., Borodin S.L., The mathematical model of the gas hydrate deposit development in permafrost. International Journal of Heat and Mass Transfer, 2018, vol. 118, pp. 455-461.

32. Hao Xu, Wen Zhou, Qian Cao, Christopher Xiao, Qiumei Zhou, Haotian Zhang, Yeyu Zhang. Differential fluid migration behaviour and tectonic movement in Lower Silurian and Lower Cambrian shale gas systems in China using isotope geochemistry. Mar. Pet. Geol., 2017, vol. 89, pp. 47-57.

33. Yintao Lu, Xiwu Luan, Fuliang Lyu, Bin Wang, Zhili Yang, Taotao Yang, Genshun Yao. Seismic evidence and formation mechanism of gas hydrates in the Zhongjiannan Basin, Western margin of the South China Sea. Marine and Petroleum Geology, 2017, vol. 84 , pp. 274-288.

34. Jinxing Dai, Yunyan Ni, Shipeng Huang, Weilong Peng, Wenxue Han, Deyu Gong, Wei Wei. Genetic types of gas hydrates in China. Petroleum Exploration and Development, 2017, vol. 44, pp. 887-898.

35. Bin Cheng, Jianbing $\mathrm{Xu}$, Zhenquan Lu, Yonghong Li, Weichao Wang, Shan Yang, Hu Liu, Ting Wang, Zewen Liao. Hydrocarbon source for oil and gas indication associated with gas hydrate and its significance in the Qilian Mountain permafrost, Qinghai, Northwest China. Mar. Pet. Geol., 2017, vol. 89, pp. 202-215.

36. Yu Hu, Min Luo, Linying Chen, Qianyong Liang, Dong Feng, Jun Tao, Shengxiong Yang, Duofu Chen. Methane source linked to gas hydrate system at hydrate drilling areas of the South China Sea: ore water geochemistry and numerical model constraints. Journal of Asian Earth Sciences, 2018, vol. 44, pp. 2-15.

37. Junli Gong, Xiaoming Sun, Li Xu, Hongfeng Lu. Contribution of thermogenic organic matter to the formation of biogenic gas hydrate: Evidence from geochemical and microbial characteristics of hydrate-containing sediments in the Taixinan Basin, South China Sea. Marine and Petroleum Geology, 2017, vol. 80, pp. 432-449.

38. Frye M., Shedd W., Boswell R. Gas hydrate resource potential in the Terrebonne Basin, Northern Gulf of Mexico. Marine and Petroleum geology, 2011, vol. 34, pp. 1-19.

Received: 18 September 2019.

\section{Information about the authors}

Alexander V. Poletaev, Cand. Sc., leading researcher Oil and Gas Institute of National Academy of Sciences of Azerbaijan.

Elena V. Poletaeva, Dr. Sc., leading researcher Oil and Gas Institute of National Academy of Sciences of Azerbaijan. 